\title{
Smart textiles biotechnology for electrocardiogram monitoring in horses during exercise on treadmill: Validation tests
}

\author{
Martina Felici ${ }^{1}$ | Mimma Nardelli ${ }^{2,3}$ | Antonio Lanatà ${ }^{4}$ | Micaela Sgorbini ${ }^{1}$ (i) | \\ Enzo Pasquale Scilingo ${ }^{2,3}$ | Paolo Baragli ${ }^{1,3}$
}

\author{
${ }^{1}$ Department of Veterinary Sciences, \\ University of Pisa, Pisa, Italy \\ ${ }^{2}$ Department of Information Engineering, \\ School of Engineering, University of Pisa, \\ Pisa, Italy \\ ${ }^{3}$ Research Center "E. Piaggio", University of \\ Pisa, Pisa, Italy \\ ${ }^{4}$ Department of Information Engineering, \\ University of Florence, Firenze, Italy

\section{Correspondence} \\ Paolo Baragli, Department of Veterinary \\ Sciences, University of Pisa, viale delle \\ Piagge 2, 56124, Pisa, Italy. \\ Email: paolo.baragli@unipi.it \\ Funding information \\ University of Pisa.
}

\begin{abstract}
Background: There are several bioengineering solutions aimed at improving human health and welfare. Smart electrodes based on textile substrates have met the growing demand for comfort, reliability, and robustness when acquiring physiological signals.

Objectives: Given the importance of good quality electrocardiograms (ECG) in equine sports medicine, this study focuses on the validation of smart textile electrodes to acquire ECG signals in horses during treadmill exercise.

Study design: The performance of the smart textile electrodes is compared with standard silver/silver chloride $(\mathrm{Ag} / \mathrm{AgCl})$ electrodes in terms of signal quality.

Methods: Five healthy Standardbred mares were fitted with two identical electronic systems for the simultaneous recording of ECGs during a standardised exercise test (SET) on a treadmill. One system was equipped with smart textile electrodes, whereas the second was equipped with standard $\mathrm{Ag} / \mathrm{AgCl}$ electrodes. The $\mathrm{Ag} / \mathrm{AgCl}$ electrodes were positioned on shaved skin with self-adhesive pads, and without (SET1) or with glue (SET2). The textile electrodes were positioned without shaving the skin. The Kurtosis $(k)$ value for each ECG trace recorded was calculated as an index of ECG signal quality.

Results: For the textile electrodes, $\mathrm{k}$ values were higher, and closer to ideal compared to $\mathrm{Ag} / \mathrm{AgCl}$ electrodes. The median values of the Signal Quality Indexes (KSQI) were higher for textile compared to $\mathrm{Ag} / \mathrm{AgCl}$ electrodes. These differences were significant in SET $2(P<.001)$, but not in SET $1(P=.08)$.

Main limitations: This study was limited to treadmill exercise that did not include a rider or harness.

Conclusions: During treadmill exercise, textile electrodes are a practical solution for collecting good quality ECG traces.
\end{abstract}

\section{KEYWORDS}

horse, ECG, kurtosis, electrodes, Signal Quality Index, motion artefacts 


\section{1 | INTRODUCTION}

Exercising ECG is an important component of clinical investigation of horses with potential cardiac arrhythmias. ${ }^{1}$ Obtaining an ECG trace of good quality during exercise is difficult because the movements of the horse often affects the ECG quality leading to motion artefacts and low stability. ${ }^{2}$ The artefacts can be generated by non-cardiac electrical potentials due to the movement between the electrodes and the skin. ${ }^{3,4}$ Skin stretch, gait movements, muscle tremor, respiratory motion, and movement of the leads ${ }^{2}$ modify the distribution of the charge at the interface between the skin and the electrode, regardless of the cause itself. ${ }^{4}$ This results in changes in the ECG during exercise, which can often lead to misinterpretation or misleadingly normal ECG waves. ${ }^{5}$

A better solution for recording ECGs in exercising horses is needed. Several bioengineering solutions have been implemented to collect human physiological signals. One of the most promising is smart textiles, which combine conductive yarn (based on stainless steel fibres) with elastane (attached to the fabric during manufacturing). Smart textiles are easy to use and comfortable; they can also be positioned without using adhesive electrodes, glue or cohesive bandages. ${ }^{6}$ The multilayer structure of the smart textiles enables an electrochemical equilibrium to be established between the skin and electrodes. This is helped by sweating and the reduction in evaporation on the body surface. The signal recording is more constant, and the quality is therefore notably improved. ${ }^{7,8}$ Smart textiles have been proposed for monitoring physiological parameters in horses, ${ }^{9}$ and have already been validated in standing horses. $^{7}$

Our hypothesis was that using smart textiles would reduce the number of motion artifacts in the equine ECG trace collected during exercise. We compared ECG traces obtained simultaneously (in an intra-subject comparison) from smart textile and common silver/silver chloride $(\mathrm{Ag} / \mathrm{AgCl})$ electrodes attached with their adhesive alone or with additional glue. ${ }^{2}$ To test the hypothesis, the Kurtosis and the kSQI (calculated based on the Kurtosis values) were used to evaluate quality of the ECG signal. Kurtosis is considered one of the best indices for the evaluation of the quality of ECG traces obtained from the leads of individual single ECG. ${ }^{10}$

\section{MATERIALS AND METHODS}

\section{1 | Animals and husbandry}

Five healthy Standardbred mares (mean age $4.8 \pm 1.1$ and weight $560.3 \pm 51.2 \mathrm{~kg}$ ) were included. They were kept in paddocks $\left(75 \times 75 \mathrm{~m}^{2}\right)$, fed with concentrate and hay and offered water ad libitum. The mares had previously been used as recipients in an embryo transfer program at the Department of Veterinary Sciences (University of Pisa, Italy). This study was conducted from January to March 2017 and none of the subjects were pregnant during the tests.

\section{2 | Experimental design}

\subsection{1 | Training period}

To accustom the horses to using the treadmill (Sato II, Sweden) and to reach an adequate level of performance, each horse underwent a training period for 1 month. The progression in speed, time and distance was modulated in relation to heart rate in order to reach 200 bpm when the horse was making maximum effort. ${ }^{11}$

\subsection{2 | Standardised exercise test}

Each horse performed two Standardised Exercise Tests (SETs) on a treadmill carried out 1 week apart. Each SET lasted 18 minutes and started with one step of 4.5 minutes walking (Walk 1), followed by three steps at increasing speeds (Trot 1, Trot 2 and Gallop), lasting 3 minutes each. The Gallop was followed by another $4.5 \mathrm{~min}$ utes walking (Walk 2). The speeds were the same for all the subjects in Walk $1(1.7 \mathrm{~m} / \mathrm{s})$, Trot $1(3.5 \mathrm{~m} / \mathrm{s})$, Trot $2(5.5 \mathrm{~m} / \mathrm{s})$ and Walk 2 $(1.7 \mathrm{~m} / \mathrm{s})$. Only one subject differed from the others in terms of the speed in Trot $2(6.5 \mathrm{~m} / \mathrm{s}$ rather than $5.5 \mathrm{~m} / \mathrm{s})$. The Gallop speeds varied amongst subjects $(8.5,9.0,10.0$ or $11.0 \mathrm{~m} / \mathrm{s})$, based on the individual heart rate and were modified to ensure each horse galloped at 200 beats $/ \mathrm{min}$.

\section{3 | Data collection}

During the two SETs, each horse was equipped with two identical ECG recording devices (Biopac). One was connected to two traditional $\mathrm{Ag} / \mathrm{AgCl}$ electrodes (3M), and the other was connected to two smart textile electrodes (Smartex Srl, Navacchio). Thus, two ECG traces were recorded simultaneously.

Recording devices were connected to the electrodes through high conductivity wires. These were sewn to the textile electrodes, thus the conductive fibres in the textile were continuous with the wires. For the $\mathrm{Ag} / \mathrm{AgCl}$ electrodes, wires were welded to a cavity button that fitted into the snap button of the electrodes. The other head of the wires was introduced into the recording devices through a plug.

The $\mathrm{Ag} / \mathrm{AgCl}$ electrodes were positioned on shaved skin using self-adhesive pads (SET 1) or with (SET 2) additional glue (Dermabond, Ethicon Inc., J\&J Medical, Somerville). The textile electrodes (rectangular, $8 \times 3 \mathrm{~cm}$ ) were horizontally positioned without shaving the skin but with alcohol applied between the skin and the electrode. Each pair of electrodes (textile and $\mathrm{Ag} / \mathrm{AgCl}$ ) produced a single lead ECG trace. The $\mathrm{Ag} / \mathrm{AgCl}$ electrodes were placed above the textile electrodes at a distance of no more than $2 \mathrm{~cm}$ (Figure 1A) in a modified base-apex configuration (Figure 1B). Electrodes were kept in place using an elastic belt fastened around the chest behind the shoulder of the horse. Between the elastic belt and the electrodes, sponges were inserted to obtain a uniform pressure. The two 
FIGURE 1 Placement of $\mathrm{Ag} / \mathrm{AgCl}$ and textile electrodes (A). Modified base apex configuration of electrodes (B)
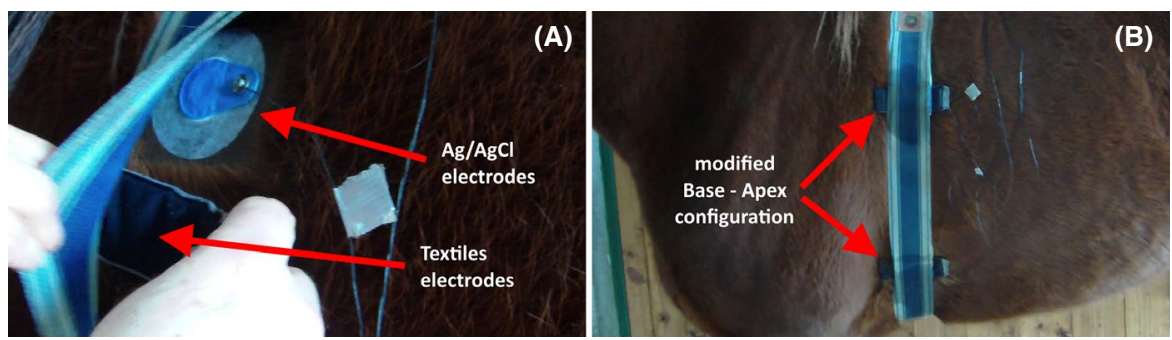

recording devices were placed in a pocket on the elastic belt and wires connecting the electrodes to the recording systems were fixed using adhesive tape.

The ECGs were acquired at a sampling frequency of $250 \mathrm{~Hz}$ (250 sample/s). The signals were transmitted wirelessly, via Bluetooth, to a mobile device. It was thus possible to assess the recording and storing process of the ECGs in real time. The ECGs were recorded on a treadmill, during 18 minutes of a standardised exercise test.

\subsection{Data analysis}

Of the total 18 minutes of recording, 15 minutes of the ECG traces were analysed, encompassing the last three minutes of Walk 1 and the first three minutes of Walk 2 thus creating five homogeneous work sessions lasting three minutes each (Walk 1, Trot 1, Trot 2, Gallop and Walk 2). Traces stored within each electronic device were downloaded to a PC for evaluation of the quality of the ECG signals. The Kurtosis $(\mathrm{k})$ values for each trace were calculated through a signal process analysis developed for humans. ${ }^{10}$ This is an index of ECG signal quality, comparing the shape of the ECG probability distribution to a Gaussian probability distribution. ${ }^{10}$ The description of the values used to categorise an ECG signal as good (sinus rhythm) or not, are reported in $\mathrm{Li}$ et al. ${ }^{12}$ As suggested by Li, good quality ECG signals have $\mathrm{k}$ values greater than $5 .{ }^{10}$ If the ECG signal is affected by artefacts, it shows a $\mathrm{k}$ value of about 5 . Interference due to power supply induces a k-value lower than $5 .^{13}$

Baseline wandering of each acquired ECG signal was removed using a Finite Impulse Response (FIR) high-pass zero phase forward-backward filtering with a cut-off frequency of $0.5 \mathrm{~Hz} .^{10,12,13}$ Then, the whole duration of each ECG signal (15 minutes) acquired from each horse in each SET, was divided into time windows of 10 seconds, overlapped by five-second intervals such that for each of the five 3 minutes standardised work sessions, a total of 3610 seconds time windows were evaluated. The k-value was then calculated for each time window. $A$ binary value $B$ was assigned to each window, as follows:

$$
\begin{aligned}
& B=1 \text { if } k \text {-value }>5 \\
& B=0 \text { if } k \text {-value } \leq 5 .
\end{aligned}
$$

The Kurtosis Signal Quality Index (KSQI) ${ }^{10,14,15}$ was calculated as follows:

$\mathrm{kSQ}=\frac{\text { number of } 10 \mathrm{~s} \text { windows where } \mathrm{B}=1}{\text { total number of } 10 \mathrm{~s} \text { windows in each session ( } 36 \text { in this study) }}$

Given the non-Gaussian distribution of samples (Shapiro-Wilk test), a two-way Friedman test was computed for each SET $(P \leq .05)$. The two vectors of $\mathrm{kSQ}$ l were used (one for $\mathrm{Ag} / \mathrm{AgCl}$ and one for textile electrodes) as the input for the statistical test. These vectors had been extracted from the equine ECGs in each experimental phase. Therefore, the number of observations made was $25(\mathrm{~N}=25)$ for each SET. The kSQI values were compared between the $\mathrm{Ag} / \mathrm{AgCl}$

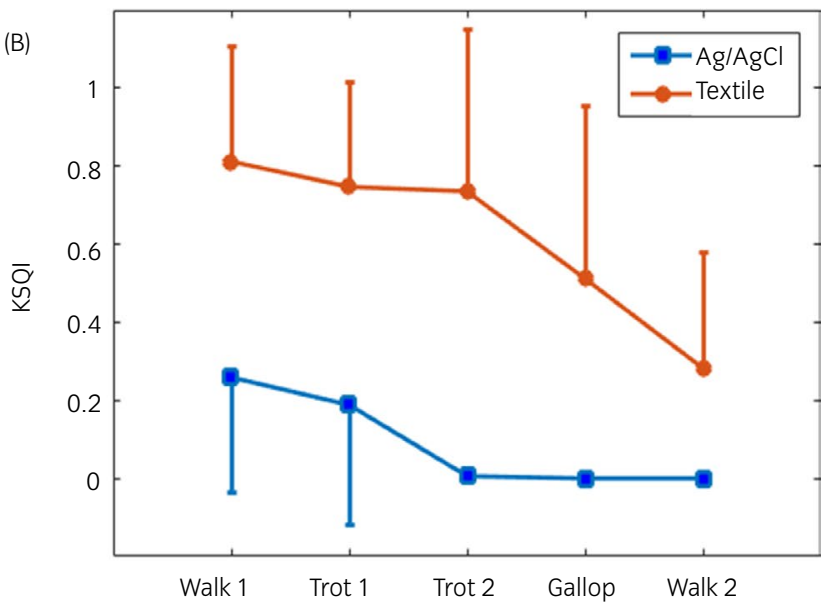

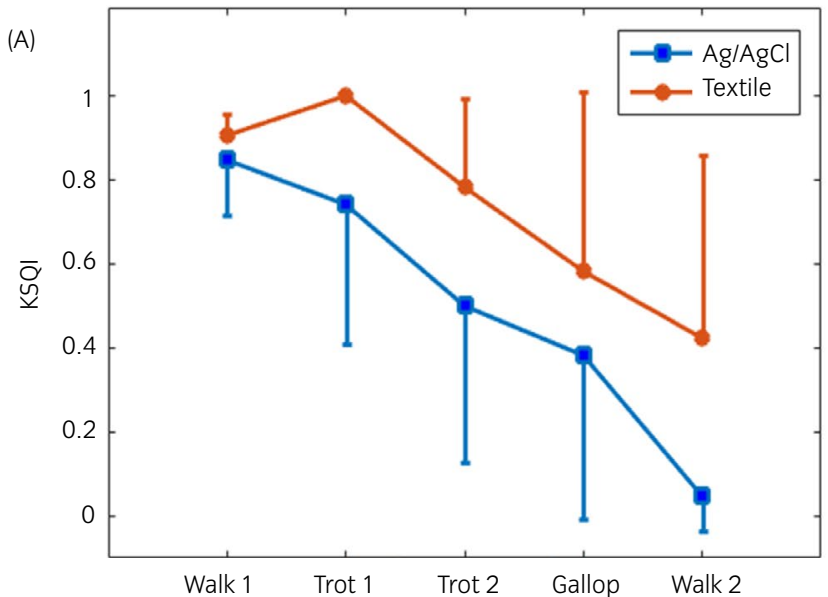

FIGURE 2 Median values and median absolute deviations (of the Kurtosis Signal Quality Index (kSQI). These values were calculated for the five work sessions (three minutes each) and in five subjects tested in two standard exercise tests. (A) With $\mathrm{Ag} / \mathrm{AgCl}$ electrodes attached without glue and (B) with $\mathrm{Ag} / \mathrm{AgCl}$ electrodes attached with glue 

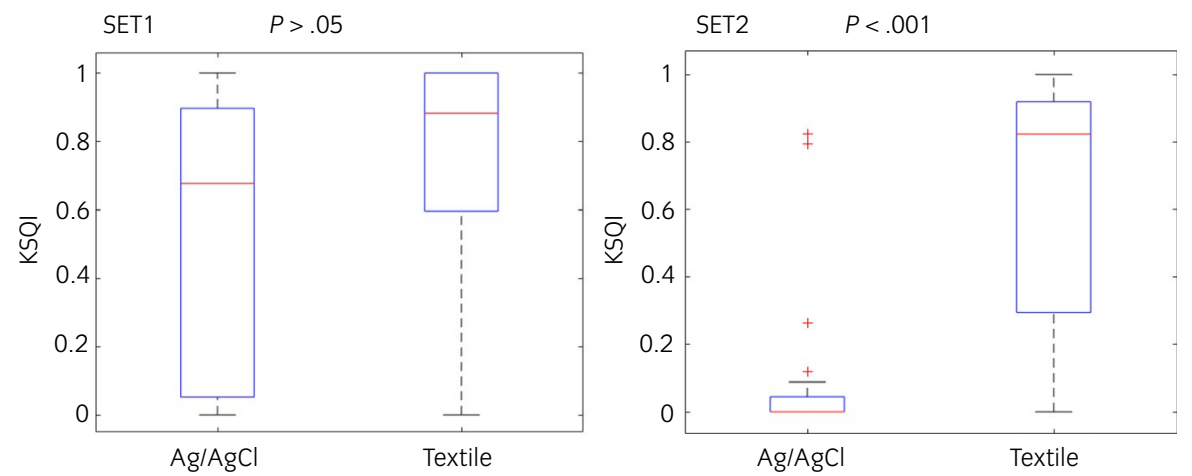

FIGURE 3 Boxplots and median values related to the range of distribution of the Kurtosis Signal Quality Index (kSQI) values for all the gaits and all the horses in the two Standard Exercise Tests (SETs) with the corresponding P-values, each referring to 25 observations). In SET 1 textile electrodes and $\mathrm{Ag} / \mathrm{AgCl}$ applied without using glue were used to simultaneously record ECG, while in SET 2 the glue was used to fix the $\mathrm{Ag} / \mathrm{AgCl}$ electrodes. The ideal value for the $\mathrm{kSQI}$ index is 1

(A)
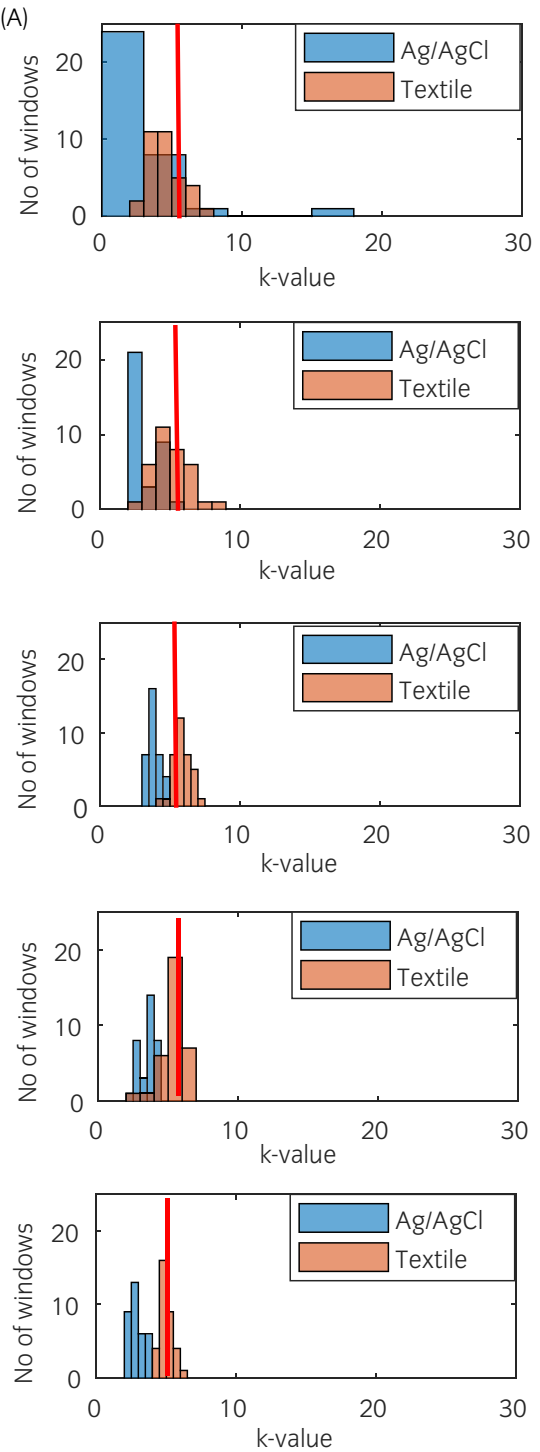

Walk 1

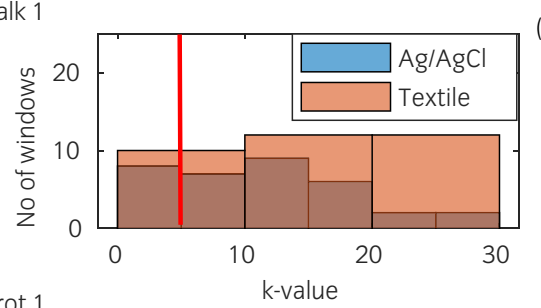

Trot 1

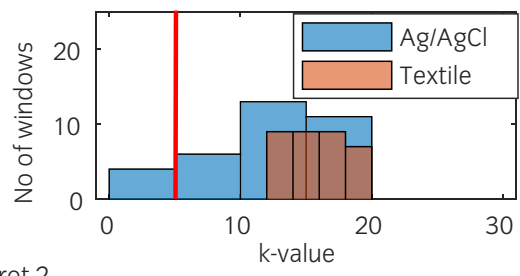

Trot 2
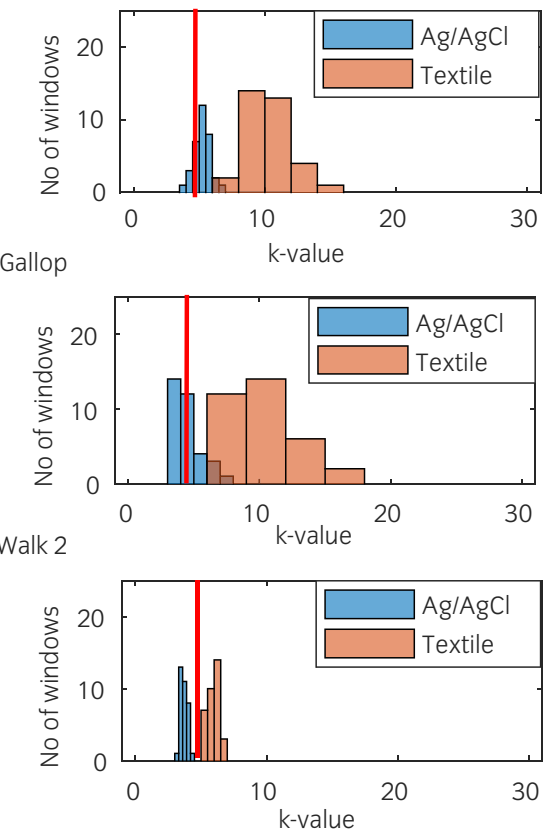

FIGURE 4 Histograms of $k$ values recorded in one subject for each three minute phase of two standardised exercise tests ( $\mathrm{A}=\mathrm{SET} 1, \mathrm{~B}=\mathrm{SET} 2$ ). The vertical axis represents the number of time windows, with the corresponding $\mathrm{k}$-value on the horizontal axis. The vertical red line indicates the k-value 5 , values above which indicate good ECG quality signal $^{11,14,15}$. The values of kurtosis for textile electrodes are over 5, while for $\mathrm{Ag} / \mathrm{AgCl}$ electrodes, they are generally below 5 electrodes and the textile electrodes, for each step (5 steps: Walk 1 , Trot 1, Trot 2, Gallop and Walk 2) and for each horse (5 horses). Our aim was to test whether there was a significant statistical difference between the quality indexes related to the two acquisition systems. All the processing tools and statistical analyses were performed with Matlab (MathWorks). 

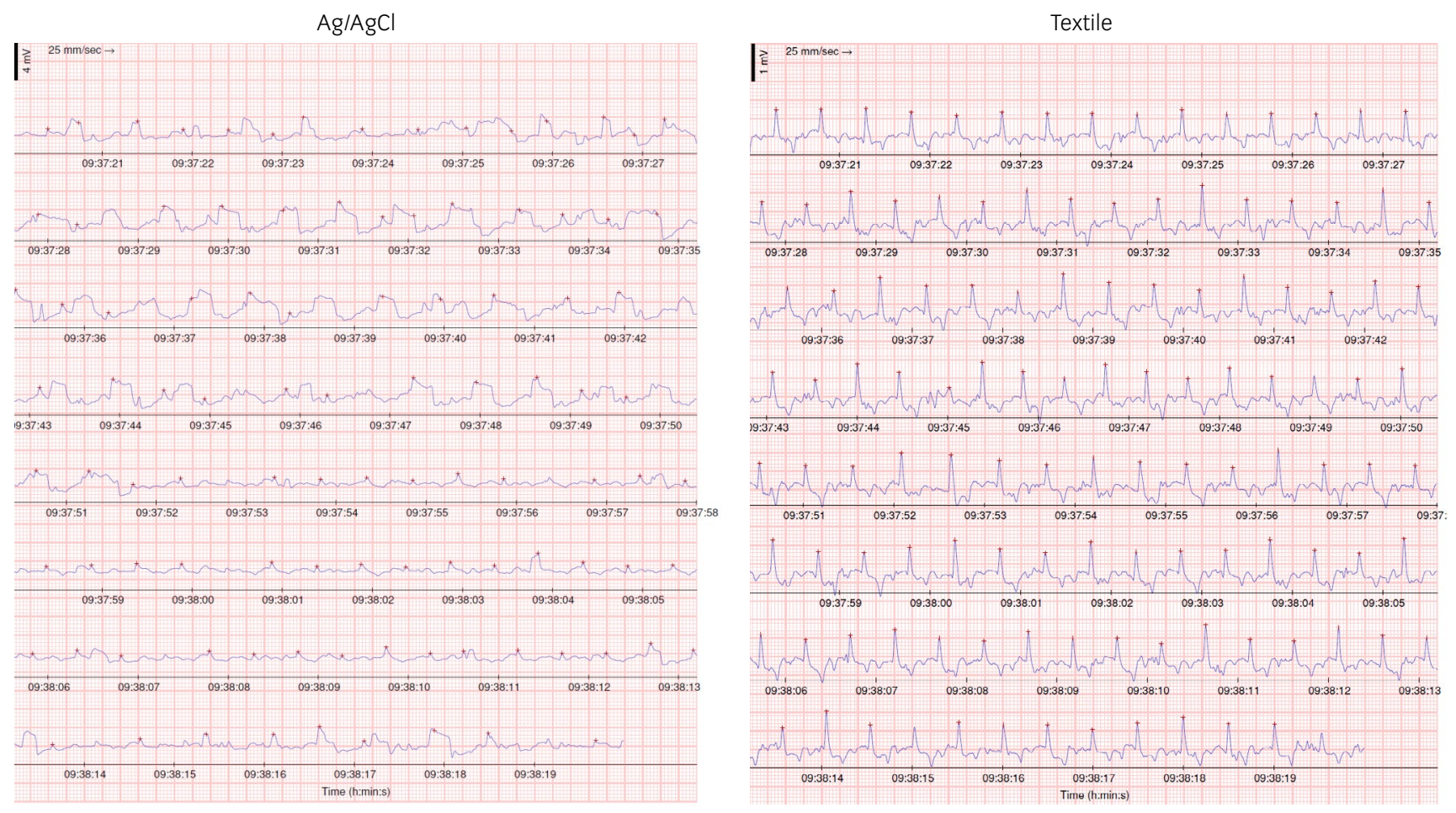

FIGURE 5 Example of ECGs acquired simultaneously using $\mathrm{Ag} / \mathrm{AgCl}$ (left), textile electrodes (right)

\section{3 | RESULTS}

Figure $2 \mathrm{~A}, \mathrm{~B}$ show that the $\mathrm{kSQ}$ I values of textile electrodes are higher than the $\mathrm{kSQ}$ of $\mathrm{Ag} / \mathrm{AgCl}$ electrodes signals, and that they are close to the optimum value of 1 during walk in the first SET. Comparing the results of the $\mathrm{Ag} / \mathrm{AgCl}$ electrodes alone, the $\mathrm{kSQ}$ l was lower in the second test (with additional glue). In general, the median KSQI values for the textile electrodes was higher than those of the $\mathrm{Ag} / \mathrm{AgCl}$ electrodes, in both SETs (Figure 3). However, in SET 1, the KSQI obtained with the $\mathrm{Ag} / \mathrm{AgCl}$ and the textile electrodes were not statistically different $(P=.08)$. In SET 2, the kSQI of the textile electrodes was significantly higher than the $\mathrm{kSQ}$ of $\mathrm{Ag} / \mathrm{AgCl}$ with glue (Table $\mathrm{S} 1$ ).

The k-value for textile electrodes was generally above the ideal value of 5 in both tests, while for $\mathrm{Ag} / \mathrm{AgCl}$, the k-value was generally less than 5. Figure 4 shows an example of data collected from one subject in each test. The histograms demonstrate that the k-values of the two electrode types became further apart as a function of speed, and the kSQI related to $\mathrm{Ag} / \mathrm{AgCl}$ decreased consistently in the last part of the experimental protocol. For visual comparison, Figure 5 shows samples of the ECG signal acquired simultaneously using the $\mathrm{Ag} / \mathrm{AgCl}$ and textile electrodes.

\section{DISCUSSION}

We used textile electrodes as an alternative method to $\mathrm{Ag} / \mathrm{AgCl}$ electrodes for ECG recording in horses during a standardised exercise on the treadmill. The comparison between $\mathrm{Ag} / \mathrm{AgCl}$ and textile electrodes was objective because the two types of electrodes were used simultaneously on the five mares enrolled in the study.
The textile electrodes are a practical solution for recording ECG traces during and with the textile electrodes, the KSQI was near to the ideal value of 1 and k-values were near to the ideal value of 5. An increase in the quality of the ECG traces obtained through textile electrodes has already been shown in horses at rest. ${ }^{7}$

In addition, two different methods for placing the $\mathrm{Ag} / \mathrm{AgCl}$ electrodes were tested. Previous studies in equine cardiology report different solutions with, ${ }^{2}$ or without, ${ }^{10}$ the application of glue on the electrode. Our results showed that the quality of the traces was reduced when the $\mathrm{Ag} / \mathrm{AgCl}$ were applied with glue compared to the textile. Therefore, using $\mathrm{Ag} / \mathrm{AgCl}$ with glue does not seem to improve the ECG quality signals which may be due to the mishandling of the electrodes when applying the glue, and the fixed state may alter the electrochemical balance of the skin. Although there was no significant statistical difference between the Signal Quality Index values (kSQI) for $\mathrm{Ag} / \mathrm{AgCl}$ and textile electrodes in SET 1, in both SETs the median value of the KSQI for textile electrodes was higher than the median value of the $\mathrm{Ag} / \mathrm{AgCl}$ electrodes.

Textile electrodes showed some practical advantages for the application in horses: they are easy to use and comfortable. The electrodes are located automatically ${ }^{6}$ without using adhesive electrodes, glue or bandages. The textile electrodes also helped the electrochemical balance, $^{7}$ thus also facilitating the continuous collection of ECG signals. The multilayer structure of the smart textile has been designed to increase the amount of sweat and reduce the rate of evaporation, thus quickly reaching an electrochemical equilibrium between the skin and electrodes. The signal quality is therefore improved and kept constant over time. ${ }^{14}$ Considering the particular characteristics of sweating in horses, ${ }^{15}$ this feature of the smart textiles may be relevant. 
The improved quality of the traces means that more advanced analysis techniques are made possible $e^{5,7}$ and ECG traces can be more accurately assessed during exercise. This may be crucial in the diagnosis of rhythm disorders and for estimating heart rate variability. ${ }^{16,17}$

The study is limited by the relatively small number of horses. Future work will focus on increasing the number of horses, which would then strengthen the statistical power of comparisons. We exercised the horses only on a treadmill, and our smart textile electrodes have not yet been tested in field settings with horses exercised under saddle or harness.

\section{1 | Conclusions}

Textile which are wearable, washable, moldable and can be applied without using glue and without having to shave the horse's coat were shown objectively to have higher signal quality compared to $\mathrm{Ag} / \mathrm{AgCL}$ electrodes. These results indicate that textile electrodes are a valid and practical alternative to classic $\mathrm{Ag} / \mathrm{AgCl}$ electrodes.

\section{ACKNOWLEDGEMENTS}

The authors thank Prof Francesco Camillo for access to the horses and stable facilities.

\section{CONFLICT OF INTEREST}

The authors declare that the research was conducted without any commercial or financial relationships that could be construed as a potential conflict of interest.

\section{AUTHOR CONTRIBUTIONS}

All authors contributed to the phases of the research. M. Felici, M Sgorbini, A. Lanatà, E.P. Scilingo and P. Baragli conceived and designed the experiments. M. Felici, M. Sgorbini, A. Lanatà and P. Baragli performed the experiments. M. Nardelli and E.P. Scilingo analysed the data. M. Nardelli, A. Lanatà and E.P. Scilingo contributed materials and analysis tools. M. Felici, M. Nardelli, A. Lanatà and P. Baragli wrote the manuscript.

\section{ETHICAL ANIMAL RESEARCH}

The Ethics Committee on Animal Experimentation of the University of Pisa approved the experimental protocol (reference No. 0010135/2018).

\section{OWNER INFORMED CONSENT}

Not applicable.

\section{DATA ACCESSIBILITY STATEMENT}

The data that support the findings of this study are available from the corresponding author upon reasonable request.

\section{ORCID}

Micaela Sgorbini iD https://orcid.org/0000-0002-0294-1803

Paolo Baragli iD https://orcid.org/0000-0001-5221-5714

\section{REFERENCES}

1. Reef VB, Bonagura J, BuhIR, McGurrin MKJ, Schwarzwald CC, van Loon $G$, et al. Recommendations for management of equine athletes with cardiovascular abnormalities. J Vet Intern Med. 2014;28(3):749-61.

2. Verheyen T, Decloedt A, De Clercq D, Deprez P, Sys SU, van Loon G. Electrocardiography in horse-part 1: how to make a good recording. Vlaams Diergen Tijds. 2010a;79(5):331-6.

3. Verheyen T, Decloedt A, De Clercq D, Deprez P, Sys SU, van Loon G. Electrocardiography in horse-part 2: how to read the equine ECG. Vlaams Diergen Tijds. 2010b;79(5):337-44.

4. de Talhouet H, Webster JG. The origin of skin-stretch-caused motion artifacts under electrodes. Physiol Meas. 1996;17(2):81.

5. Lanata A, Guidi A, Baragli P, Valenza G, Scilingo EP. A novel algorithm for movement artifact removal in ECG signals acquired from wearable systems applied to horses. PLoS One. 2015;10(10):e0140783.

6. Lymberis A, Paradiso R. Smart Fabrics and Interactive Textile Enabling Wearable Personal Application: R\&D State of Art and Future Challenges. Ann Int IEEE Eng Med Bio 30th, August 20-24, Vancouver, Canada, 5270-5273. 2008.

7. Guidi A, Lanata A, Valenza G, Scilingo EP, Baragli P. Validation of smart textile electrodes for electrocardiogram monitoring in free-moving horses. J Vet Behav. 2017;17:19-23.

8. Scilingo EP, Lorussi F, Mazzoldi A, De Rossi D. Strain-sensing fabrics for wearable kinaesthetic-like systems. IEEE Sens J. 2003;3(4):460-7.

9. McGreevy PD, Sundin M, Karlsteen M, Berglin L, Ternstrom J, Hawson L, et al. Problems at the human-horse interface and prospect for smart textile solutions. J Vet Behav. 2014;9:34-42.

10. Clifford GD, Behar J, Li Q, Rezek I. Signal quality indices and data fusion for determining clinical acceptability of electrocardiograms. Physiol Meas. 2012;33:1419-33.

11. Allen KJ, Young LE, Franklin SH. Evaluation of heart rate and rhythm during exercise. Equine Vet Educ. 2016;28(2):99-112.

12. Li Q, Mark RG, Clifford GD. Robust heart rate estimation from multiple asynchronous noisy sources using signal quality indices and a Kalman filter. Physiol Meas. 2008;29:15-32.

13. Behar J, Oster J, Li Q, Clifford GD. ECG signal quality during arrhytmia and its application to false alarm reduction. IEEE T Bio Med Eng. 2013;60(6):1660-6.

14. Scilingo EP, Gemignani A, Paradiso R, Taccini N, Ghelarducci B, De Rossi D. Performance evaluation of sensing fabrics for monitoring physiological and biomechanical variables. IEEE Trans Inf Technol Biomed. 2005;9:345-52.

15. Scott CM, Marlin DJ, Schroter RC. Quantification of the response of equine apocrine sweat glands to b2-adrenergic stimulation. Equine Vet J. 2001;33:605-12.

16. Bowen IM. Ambulatory electrocardiography and heart rate variability. In: Marr CM, Bowen IM, editors. Cardiology of the horse. 2nd ed. London, UK: Saunders, p. 127-37.

17. Physick-Sheard PW, Marlin DJ, Thornhill R, Schroter RC. Frequency domain analysis of heart rate variability in horses at rest and during exercise. Equine Vet J. 2000;32(3):253-62.

\section{SUPPORTING INFORMATION}

Additional supporting information may be found online in the Supporting Information section.

How to cite this article: Felici M, Nardelli M, Lanatà A, Sgorbini M, Pasquale Scilingo E, Baragli P. Smart textiles biotechnology for electrocardiogram monitoring in horses during exercise on treadmill: Validation tests. Equine Vet J. 2020;00:1-6. https://doi.org/10.1111/evj.13296 